\title{
Steroid-mediated liver steatosis is CD1d-dependent while steroid-induced liver necrosis, inflammation, and metabolic changes are CD1d-independent: A dichotomy between steatosis and steatohepatitis
}

\section{Tomer Adar \\ Hadassah Medical Center \\ Ami Ben Ya'acov \\ Hadassah Medical Center \\ Shabat Shabat \\ Hadassah Medical Center \\ Meir Mizrahi \\ Hadassah Medical Center \\ Lida Zolotarov \\ Hadassah Medical Center \\ Yoav Lichtenstein \\ Hadassah Medical Center}

Yaron Ilan ( $\square$ ilan@hadassah.org.il)

Hadassah Medical Center https://orcid.org/0000-0003-0802-1220

\section{Research Article}

Keywords: Steroids, NKT cells, NAFLD, NASH, glycosphingolipids, glucocerebroside

Posted Date: April 21st, 2021

DOI: https://doi.org/10.21203/rs.3.rs-339739/v1

License: (c) (1) This work is licensed under a Creative Commons Attribution 4.0 International License. Read Full License 


\section{Abstract}

Glucocorticoids have been implicated in the pathogenesis of all stages of non-alcoholic fatty liver disease (NAFLD). Natural killer T cells play a role in the pathogenesis of NAFLD and in the response to steroids. The aim of the present study was to determine the role of CD1d in steroid-mediated metabolic derangement and the steroid-protective effect of glycosphingolipids.

Methods: Ten groups of mice were studied. Steroids were orally administered to C57BL/6 mice to assess the therapeutic effect of $\beta$-glucsylceramide (GC) on the development of steroid-mediated liver damage and metabolic derangements. The role of CD1d in the pathogenesis of steroid-induced liver damage, and in mediating the hepatoprotective effect of GC were studied in $\mathrm{CD} 1 \mathrm{~d}^{-/-}$mice.

Results: A model of oral administration of steroids was established, resulting in insulin resistance, hyperinsulinemia, hypertriglyceridemia, liver steatosis, and hepatocellular injury. Steroid administration to $\mathrm{CD} 1 \mathrm{~d}^{-/-}$mice was associated with hyperglycemia and hypertriglyceridemia. However, $\mathrm{CD} 1 \mathrm{~d}^{-/-}$mice were relatively resistant to steroid-induced steatosis. GC treatment alleviated steroid-associated metabolic derangements and liver injury independent of CD1d expression.

Conclusion: A steroid-mediated model of NAFLD and metabolic derangements was established in which steroid-mediated steatosis was CD1d-dependent while steroid-induced liver necrosis, inflammation, and metabolic changes were CD1d-independent, further supporting a dichotomy between steatosis and steatohepatitis in NAFLD.

\section{Introduction}

Glucocorticoid administration inhibits mitochondrial beta-oxidation and lipid beta-peroxidation enzymes, leading to hepatic steatosis. Glucocorticoids inhibit medium- and short-chain acyl-CoA dehydrogenation and hepatic lipid secretion ${ }^{1}$. In addition, corticosteroids promote de novo fatty acid synthesis by stimulating lipogenic enzymes including fatty acid synthase, acetyl-CoA carboxylase, and 11 betahydroxysteroid dehydrogenase type 1 in the liver $^{2}$. Glucocorticoids increase hepatic phosphatidate phosphatase (PAP1) activity, which promotes the storage of fatty acids as triacylglycerols (TAGs). TAGs are consequently used for beta-oxidation or very low-density lipoprotein (VLDL) secretion ${ }^{3}$.

Glucocorticoids are catabolic in nature, leading to the delivery of energy substrates during stress $s^{4,5}$. Steroids play a role in the development of diet-induced obesity by modulating fasting triglyceride metabolism ${ }^{6}$. During the fasting state, glucocorticoids mobilize lipids, thus increasing fatty acid delivery, and in the fed state they promote lipid accumulation ${ }^{7}$. Steroids augment the levels of circulating fatty acids by increasing the caloric and dietary fat intake and the hydrolysis of circulating triglycerides by lipoprotein lipase activity ${ }^{4}$. This may be associated with ectopic fat distribution in the liver, muscle, and central adipocytes. 
Excessive action of cortisol and fatty acids contributes to the development of insulin resistance and its associated pathologies ${ }^{8}$. An imbalance of the hypothalamic-pituitary-adrenal-axis exacerbates the effects of cortisol ${ }^{8}$. Fatty acids and cortisol induce insulin resistance, augmenting lipogenesis in the liver and hepatic secretion of glucose and $\mathrm{VLDL}^{9}$. In the presence of high insulin concentrations, cortisol promotes the deposition of energy and leads to obesity ${ }^{8}$. Lowered glucose metabolism is associated with altered fatty acid supply, composition, and utilization, as well as with "lipotoxicity", which further contributes to steroid-mediated liver damage ${ }^{10}$.

Glucocorticoids were proposed to play a role in the pathogenesis of all stages of non-alcoholic fatty liver disease (NAFLD) by affecting both the liver and the adipose tissue ${ }^{7}$. Both microvesicular steatosis and ultrastructural mitochondrial lesions have been reported ${ }^{1}$. Chronic administration of glucocorticoids and a high-fat diet (HFD) independently induce insulin resistance, abdominal obesity, and NAFLD. In a rodent model, the combination of glucocorticoids and an HFD resulted in central obesity, insulin resistance, increased liver lipid content, and fibrosis ${ }^{11}$. The liver damage was independent of inflammation ${ }^{11}$ and of the anti-inflammatory effect of steroids ${ }^{12}$.

The use of intravenous glucocorticoids as a treatment for various steroid-responsive diseases may be associated with liver toxicity. An increase in anabolic androgenic steroid-mediated hepatotoxicity is a major public health problem ${ }^{13}$. Both hepatocellular and cholestatic features have been described. Statins, for example, when concomitantly employed with methylprednisolone, lead to liver dysfunction ${ }^{14}$. Natural killer T (NKT) cells are a unique subset of cells that are mostly abundant in murine and human livers. NKT cells are divided into type 1 and type 2 according to their dependence on the interaction with CD1d, a non-polymorphic glycolipid antigen presenting molecule. NKT cells modulate hepatic inflammation through CD1d recognition in conjunction with glycolipid antigen and they were found to have a protective role in the pathogenesis of NAFLD ${ }^{15}$, and in the response to steroids ${ }^{16,17}$. Glycosphingolipids (GSLs) have been described to exert a hepatoprotective effect in animal models by alleviating liver inflammation via an NKT cell-mediated effect ${ }^{18-28}$. The aim of the present study was to determine the role of CD1d in steroid-mediated liver injury, metabolic derangement, and the steroid-protective effect of GSLs.

\section{Methods}

Animals: 10-week-old male C57BL/6 mice were purchased from Harlan Laboratories (Jerusalem, Israel). $\mathrm{CD}_{1} \mathrm{~d}^{-/-}$mice were kindly provided by Dr. Luc Van Kaer, Department of Microbiology and Immunology Medical Center North, Vanderbilt University School of Medicine, Nashville, TN. Mice were maintained in the animal core of the Hadassah-Hebrew University Medical School. All mice were administered standard laboratory chow and water ad libitum and maintained in a 12-hour light/dark cycle. The animal experiments were carried out according to the guidelines of the Hebrew University-Hadassah Institutional Committee for Care and Use of Laboratory Animals and with the committee's approval. 
Induction of liver and metabolic damage and GSL treatment: Metabolic and liver damage were induced by oral administration of Dexamethasone ( $0.5 \mathrm{mg} /$ day). b-glucosylceramide (GC) was purchased from Avanti Polar Lipids (Alabaster, AL, USA), dissolved in a vehicle of a mixture of 30\% Cremophor EL (Sigma, Rehovot, Israel) and ethanol (1:1) in PBS, and administered orally at a dose of $15 \mathrm{mg} /$ day.

Study design. Mice in all groups were followed to determine the effect of steroids and GC on metabolic derangements and liver damage. The graphs are representative of three separate experiments in which similar results were obtained

\section{Experimental groups:}

Ten groups of mice were studied in three experiments (Table 1). In the first part of the study, three groups of C57BL/ 6 mice were followed for eight weeks to assess the preventive effect of GC on the development of steroid-mediated liver damage. Group A received vehicle alone, Group B received dexamethasone, and group $C$ received a combination of dexamethasone and GC. To determine the therapeutic effect of GC on existing damage, four groups of C57BL/ 6 mice were studied in the second part of the study. Groups D to $F$ were identical to groups $A$ to $C$ in the first part of the study. Mice in group $D$ received vehicle alone, group $\mathrm{E}$ received dexamethasone, group $\mathrm{F}$ received a combination of dexamethasone and GC, mice in group $\mathrm{G}$ received dexamethasone for 6 weeks followed by two weeks of administration of dexamethasone in combination with GC. To determine the involvement of CD1d in steroid-induced liver damage and the steroid-protective effect of $\mathrm{GC}$, we used $\mathrm{CD} 1 \mathrm{~d}^{-/-}$mice were for the third part of the study: Mice in group $\mathrm{H}$ received vehicle alone, group I received dxamethasone, and group $\mathrm{J}$ received a combination of dexamethasone and GC for eight weeks.

Fasting serum glucose and insulin levels. Fasting serum glucose levels were measured using a standard glucometer. Serum insulin levels were determined using a commercially available ELISA kit (Mercodia AB; Uppsala, Sweden), according to the manufacturer's instructions.

Glucose tolerance test (GTT). A glucose tolerance test (GTT) was performed by oral administration of glucose $(1.25 \mathrm{~g} / \mathrm{kg})$ with repeated glucose level tests from the tail vein using a standard glucometer.

Liver enzymes and triglyceride levels. Serum aspartyl transaminase (AST), alanine aminotransferase (ALT), and serum triglycerides were measured using commercially available sticks by a Reflovet Plus analyser (Roche Diagnostics, GmbH, Mannheim, Germany).

Histological Examination. A portion of each excised liver from all mice in all groups was formalin-fixed. Tissue samples were embedded in paraffin and $5-\mu \mathrm{m}$ sections were cut. Sections were stained with hematoxylin and eosin (H\&E) and read by a blinded pathologist.

Hepatic triglycerides content. Accumulation of intrahepatic triglycerides (TGs) was quantified using a modification of the Folch method ${ }^{29}$. Triglycerides were extracted by a GPO-Trinder kit (Sigma, Rehovot, 
Israel), then quantified by a spectrophotometer, and levels were normalized to the protein content in the homogenate.

Serum cytokine levels. Serum levels of IL10 and IL 17 were measured using a commercially available sandwich ELISA kit (Quantikine, R\&D Systems, MN, USA).

Statistical analysis. Statistical analysis was performed using Student's $t$-test. A $p$ value less than 0.05 was considered significant.

\section{Results}

\section{Steroid treatment induced insulin resistance, hyperlipidemia, and liver damage}

Figure 1 shows the effect of steroids on metabolic parameters and liver damage. Oral administration of dexamethasone resulted in insulin resistance manifested by increased fasting serum glucose levels (Figure 1A) and increased GTT as shown by the study curves (Figure 1B), by the calculated areas under the curve (AUC) (Figure 1C), and by increased serum insulin levels (Figure 1D). Steroid treatment also increased serum triglyceride levels (Figure 1E). The effect of steroids on insulin and lipid metabolism was associated with a significant hepatocellular injury manifested by an increase in ALT and AST serum levels (Figure 1F).

\section{Oral administration of b-Glucosylceramide prevented the steroid-mediated metabolic and liver damage}

Co-administration of GC and steroids prevented the increase in fasting glucose levels (Figure 1A), postprandial hyperglycemia (Figures 1B and 1C), hyperinsulinemia (Figure 1D), hypertriglyceridemia (Figure $1 \mathrm{E})$, and the increase in liver enzymes (Figure 1F), signifying its protective effect against steroid-mediated metabolic and hepatic damage.

\section{Oral administration of b-Glucosylceramide alleviated steroid-mediated metabolic and liver damage}

The aim of the second part of the study (Figure 2) was to determine the therapeutic effect of orally administering GC following the induction of steroid-mediated metabolic and liver damage. GC therapy was associated with a reduction of fasting plasma glucose levels on week eight (Figure 2A), improvement in the GTT (Figure 2B), and reduction of serum insulin levels (Figure 2C). These effects were comparable to the protective effect of GC when administered for prevention (group F vs group G, prevention vs. treatment, respectively). Similarly, GC therapy alleviated steroid-mediated hypertriglyceridemia (Figure 2D) and liver injury by reducing both ALT and AST serum levels (Figure 2E). Histological analysis showed a reduction in ballooning and cell apoptosis (Figure 2F). However, GC had no effect on total liver triglyceride content in WT mice (Figure 2G). GC therapy was associated with increased in IL10 and IL17 serum levels, suggesting an effect on the systemic immune milieu in this system (Figure 2H). 


\section{CD1d-dependence of liver steatosis and CD1d-independence of the steroid-mediated damage and therapeutic effect of GC}

The aim of the third part of the study (Figure 3) was to determine the involvement of NKT cells in the steroid-mediated damage and the therapeutic effect of GC. Steroid administration in CD1 $\mathrm{d}^{-/}$mice was associated with significant insulin resistance manifested by increase fasting serum glucose levels (Figure 3A), and increased GTT (Figure 3B), along with hypertriglyceridemia (Figure 3C). Administration of steroids to $C D 1 \mathrm{~d}^{-/-}$mice was associated with significant liver damage manifested by increased liver enzymes (Figure 3D) and necrosis as seen by histological examination (Figure 3E). However, CD $1 \mathrm{~d}^{-/-}$mice were relatively resistant to the development of liver steatosis as manifested by the absence of an increase in liver TG content following steroid administration (Figure 3F).

The protective effect of GC was independent of CD1d. GC treatment alleviated steroid-mediated hyperglycemia (Figures 3A and 3B) and hypertriglyceridemia (Figure 3C). GC treatment prevented steroidmediated liver damage as manifested by a decrease in liver enzymes (Figure 3D). Histologically, GC treatment alleviated steroid-mediated hepatocyte ballooning, cell apoptosis, and steatosis. Interestingly, GC treatment significantly reduced the triglyceride content (Figure 3E), an effect that was not observed in WT mice.

\section{Discussion}

The present study established a model of oral steroid administration for eight weeks to simulate the insulin resistance and the associated liver injury which is similar to that noted in patients with metabolic syndrome. Steroid-treated mice exhibited insulin resistance, hypertriglyceridemia, and hepatocellular injury. These results support previously published data on the effect of steroids on the liver ${ }^{7,11}$. CD $1 \mathrm{~d}^{-/-}$ mice were used for determining the potential role of CD1d in mediating steroid damage. The steroidassociated hyperglycemia and hypertriglyceridemia were independent of CD1d, however, CD1d ${ }^{-/-}$mice were relatively resistant to steroid-induced steatosis. The relative resistance to steatosis was independent of the steroid-induced liver damage in these mice manifested by the increased liver enzymes and hepatocytes necrosis on histology. The present study supports a role for CD1d in the development of steatosis per se, while not preventing the steroid-mediated hepatocyte damage or metabolic derangements. These results suggest a partial role for the activation of CD1d-positive cells in NAFLD development.

Hepatic steatosis is associated with activation of the innate immune system ${ }^{30}$. Leptin-deficient $o b / o b$ mice exhibit metabolic and inflammatory features that mimic human NAFLD ${ }^{31-34}$. These mice exhibit a decrease in liver NKT cells ${ }^{35,36}$, suggesting that these lymphocytes may regulate the development of NAFLD ${ }^{30}$. In this model, leptin deficiency modulates inflammation independently of hepatic steatosis. An obese/steatosis HFD model, associated with both weight gain and hepatic steatosis, is also associated with a reduction in liver NKT cells ${ }^{30,3738}$. These findings can be interpreted as a lack of a full protective 
effect of NKT deficiency, wherein a decrease of NKT cells is associated with greater damage, independent of steatosis. These observations may also result from post activation-mediated cell death, wherein NKT activation leads to greater steatohepatitis and a subsequent decrease in NKT cells ${ }^{27}$. Endogenous IL-12 released by Kupffer cells (KC) in obesity ${ }^{30} 39$, may promote the release of IFN- $\gamma$ by activated NKT cells, with a subsequent decrease of intrahepatic NKTs ${ }^{30}, 40$. Dietary fatty acids were also linked to hepatic NKT cell deficiency ${ }^{41}$, showing that dietary saturated fatty acids (SFA) or monounsaturated fatty acids (MUFA), but not polyunsaturated fatty acids (PUFA), deplete liver NKT lymphocytes ${ }^{30,41}$.

Obesity is characterized by low-grade systemic inflammation and the adipocyte-macrophage interaction contributes to its development ${ }^{42,43}$. An interplay between insulin resistance and inflammation is thought to contribute to the development of hepatic steatosis and steatohepatitis. Anti-inflammatory agents are beneficial in animal models and in patients with $\mathrm{NASH}^{25,44}$. The results of the present study support the lack of a direct relationship between steroid-induced metabolic changes and hepatocyte injury and steatosis, implying a pathogenesis dichotomy between steatosis and liver damage in NAFLD. NAFLD comprises a spectrum from pure steatosis to NASH. The present data suggests that the mechanism that induces liver damage in NASH may be independent of steatosis, and can further explain the liver damage in lean NASH patients ${ }^{45}$, and the fact than the majority of patients with simple steatosis do not develop $\mathrm{NASH}^{46}$.

GC has been shown to act as liver-protecting agent, preventing drug-induced liver injury (DILI) of several hepatotoxic drugs ${ }^{18}$. In the present study, administering GC both prevented and treated the steroidmediated liver damage as observed by a reduction of ALT and AST serum levels and a decrease in the levels of ballooning and apoptosis upon histological examination. GC alleviated steroid-induced hyperglycemia, hyperinsulinemia, and hypertriglyceridemia. The protective effect of GC was independent of CD1d. However, while CD1d ${ }^{-/-}$mice showed less steroid-mediated steatosis, GC treatment exerted a more profound effect on reducing steatosis in these mice as compared with WT mice. These data support a dichotomy in GC function for reducing steroid-mediated steatosis, which is prevented by CD1d, which was not seen for the effect of GC on the hepatocellular damage.

Manipulations of NKT cells are beneficial in the settings of diabetes, fatty liver disease, and NASH in animal models $s^{16-20,39-41,47-50}$ and in humans ${ }^{23}$. GC treatment exerts a fat-reducing effect in animal models and humans ${ }^{23,33}$. The present data support CD1 $\mathrm{d}^{+}$lymphocyte plasticity which underlines the differences noted between the different models. NKT cells were shown to be either useful or associated with liver damage under different conditions in an environment- and/or disease-dependent manner 47,48 . GC treatment alleviates immunologically incongruous disorders, suggesting it is associated with a "fine tuning" of immune responses by altering NKT-plasticity ${ }^{48,49}$, and this effect may be CD1d-mediated ${ }^{50,51}$. The CD1d-independence of the GC-fat reduction effect under steroid therapy in the present study may also suggest an alternative CD1d-independent liver protective effect of GC. A CD1d evading mechanism, similar to the one described for infectious agents may also play a role under these conditions ${ }^{52}$. 
Interleukin-1 is an important target of steroid therapy ${ }^{53}$, and promotes the inflammation in $\mathrm{NASH}^{51}$; a possible link via IL1 and GC may explain these findings.

GC therapy has been associated with increased IL10 and IL17 serum levels, suggesting its effect on the systemic immune system. Serum IL-10 levels are significantly lower in patients with NASH ${ }^{54}$, while IL-17 exacerbates hepatic steatosis and inflammation in NAFLD ${ }^{55}$. As only two cytokines were measured in the present study, an overall alteration of the cytokine milieu in an anti-inflammatory direction may underlie the noted beneficial clinical effect. Future studies will need to dissect the subsets of cells mediating these effects.

In conclusion, our study established a model of NAFLD and metabolic derangements by steroid administration for eight weeks in which the steroid-mediated steatosis was CD1d-dependent while steroid-induced liver necrosis and inflammation, insulin resistance, and hyperlipidemia were CD1dindependent. The results further support a pathogenesis dichotomy between steatosis and steatohepatitis in NAFLD. CD1 $\mathrm{d}^{-/-}$mice were relatively resistant to steroid-induced steatosis, suggesting that NKT cells play a role in fat accumulation. The data further support the lack of a direct association between steatosis per se and liver damage. The beneficial effect of GC to alleviate the steroid inducedmetabolic changes and liver damage were independent of CD1d, while CD1d prevented the ability of GC to reduce steroid-mediated steatosis. Overall, the data supports the potential use of GC as both a liver protector to be used with steroid therapy and as drug to treat NAFLD. Our results also support the need for a combination therapy in patients with NASH wherein both inflammatory and metabolic pathways need to be targeted ${ }^{56}$. 


\begin{tabular}{|c|c|c|c|}
\hline Group & Mice & Treatment & Duration (weeks) \\
\hline A & C57BL/6 & Vehicle & 8 \\
\hline B & $\mathrm{C} 57 \mathrm{BL} / 6$ & Dexamethasone & 8 \\
\hline C & C57BL/6 & Dexamethasone $+\beta$-glucosylceramide & 8 \\
\hline D & C57BL/6 & Vehicle & 12 \\
\hline $\mathrm{E}$ & $\mathrm{C} 57 \mathrm{BL} / 6$ & Dexamethasone & 12 \\
\hline $\mathrm{F}$ & $\mathrm{C} 57 \mathrm{BL} / 6$ & $\beta$-glucosylceramide & 12 \\
\hline G & $\mathrm{C} 57 \mathrm{BL} / 6$ & $\begin{array}{l}\text { Dexamethasone for } 6 \text { weeks followed by } \\
\text { Dexamethasone }+\beta \text {-glucosylceramide additional } 2 \\
\text { weeks }\end{array}$ & $\begin{array}{l}6 \text { weeks for induction } \\
\text { and } 2 \text { weeks for } \\
\text { treatment }\end{array}$ \\
\hline $\mathrm{H}$ & CD1d-/- & Vehicle & 8 \\
\hline I & CD1d-/- & Dexamethasone & 8 \\
\hline $\mathrm{J}$ & CD1d-/- & Dexamethasone $+\beta$-glucosylceramide & 8 \\
\hline
\end{tabular}

\section{Declarations}

*Ethics approval and consent to participate: Ethical committee approval was received *Consent for publication: Yes

*Availability of data and materials: Enclosed as supplementary file

*Competing interests: None

*Funding: NA

*Authors' contributions: Tomer Adar ${ }^{1,2 \star}$, Ami Ben Ya'acov ${ }^{1}{ }^{2} \star$, Yehudit Shabat ${ }^{1}$, Meir Mizrahi ${ }^{1}$, Lida Zolotarov ${ }^{1}$, Yoav Lichtenstein ${ }^{1}$, designed and conducted the study and analyzed the data and wrote the paper. Yaron Ilan ${ }^{1}$. Designed the study and wrote the paper.

*Acknowledgements: NA

\section{Abbreviations}

NAFLD: non-alcoholic fatty liver disease; GSLs: glycosphingolipids; NKT: natural killer T cells; PAP1: phosphatidate phosphatase; TAGs: triacylglycerols; HFD: high-fat diet; GC: b-glucosylceramide; GTT: glucose tolerance test; AST: aspartyl transaminase; ALT: alanine aminotransferase; TGs: triglycerides; NASH: non-alcoholic steatohepatitis; KC: Kupffer cells; IFN-Y: Intereferon gamma; SFA: saturated fatty acids; MUFA: monounsaturated fatty acids; PUFA: polyunsaturated fatty acids; DILI: drug-induced liver injury. 


\section{References}

1. Letteron, P., N. Brahimi-Bourouina, and M. A. Robin, et al. 1997. Glucocorticoids inhibit mitochondrial matrix acyl-CoA dehydrogenases and fatty acid beta-oxidation. Am J Physio/272: G1141-G1150.

2. Jia, Y., N. Viswakarma, and T. Fu, et al. 2009. Conditional ablation of mediator subunit MED1 (MED1/PPARBP) gene in mouse liver attenuates glucocorticoid receptor agonist dexamethasoneinduced hepatic steatosis. Gene Expr 14: 291-306.

3. Manmontri, B., M. Sariahmetoglu, and J. Donkor, et al. 2008. Glucocorticoids and cyclic AMP selectively increase hepatic lipin-1 expression, and insulin acts antagonistically. J Lipid Res 49: 1056-1067.

4. Peckett, A. J., D. C. Wright, and M. C. Riddell. 2011. The effects of glucocorticoids on adipose tissue lipid metabolism. Metabolism 60: 1500-1510.

5. Macfarlane, D. P., S. Forbes, and B. R. Walker. 2008. Glucocorticoids and fatty acid metabolism in humans: fuelling fat redistribution in the metabolic syndrome. J Endocrinol 197: 189-204.

6. Mantha, L., E. Palacios, and Y. Deshaies. 1999. Modulation of triglyceride metabolism by glucocorticoids in diet-induced obesity. Am J Physiol 277: R455-R464.

7. Woods, C. P., J. M. Hazlehurst, and J. W. Tomlinson. 2015. Glucocorticoids and non-alcoholic fatty liver disease. J Steroid Biochem Mol Biol 154: 94-103.

8. Brindley, D. N. 1995. Role of glucocorticoids and fatty acids in the impairment of lipid metabolism observed in the metabolic syndrome. Int J Obes Relat Metab Disord 19 (Suppl 1): S69-S75.

9. Kneeman, J. M., J. Misdraji, and K. E. Corey. 2012. Secondary causes of nonalcoholic fatty liver disease. Therap Adv Gastroenterol 5: 199-207.

10. Qi, D., and B. Rodrigues. 2007. Glucocorticoids produce whole body insulin resistance with changes in cardiac metabolism. Am J Physiol Endocrinol Metab 292: E654-E667.

11. D'Souza, A. M., J. L. Beaudry, and A. A. Szigiato, et al. 2012. Consumption of a high-fat diet rapidly exacerbates the development of fatty liver disease that occurs with chronically elevated glucocorticoids. Am J Physiol Gastrointest Liver Physiol 302: G850-G863.

12. Rebolledo, R. A., B. Liu, and M. Z. Akhtar, et al. 2015. Steroid Anti-Inflammatory Effects Did Not Improve Organ Quality in Brain-Dead Rats. Biomed Res Int 2015: 207534.

13. Robles-Diaz, M., A. Gonzalez-Jimenez, and I. Medina-Caliz, et al. 2015. Distinct phenotype of hepatotoxicity associated with illicit use of anabolic androgenic steroids. Aliment Pharmacol Ther 41: 116-125.

14. Covelli, D., G. Vannucchi, and I. Campi, et al. 2015. Statins may increase the risk of liver dysfunction in patients treated with steroids for active graves' orbitopathy. J Clin Endocrinol Metab 100: 17311737.

15. Tajiri, K., and Y. Shimizu. 2012. Role of NKT Cells in the Pathogenesis of NAFLD. Int J Hepato/2012: 850836. 
16. Hodge, G., and S. Hodge. 2016. Steroid Resistant CD8(+)CD28(null) NKT-Like Pro-inflammatory Cytotoxic Cells in Chronic Obstructive Pulmonary Disease. Front Immunol 7: 617.

17. Kwon, H. J., Y. S. Won, and O. Park, et al. 2014. Opposing effects of prednisolone treatment on T/NKT cell- and hepatotoxin-mediated hepatitis in mice. Hepatology 59: 1094-1106.

18. Mizrahi, M., T. Adar, and G. Lalazar, et al. 2018. Glycosphingolipids Prevent APAP and HMG-CoA Reductase Inhibitors-mediated Liver Damage: A Novel Method for "Safer Drug" Formulation that Prevents Drug-induced Liver Injury. J Clin Transl Hepatol 6: 127-134.

19. Zigmond, E., O. Tayer-Shifman, and G. Lalazar, et al. 2014. beta-glycosphingolipids ameliorated nonalcoholic steatohepatitis in the Psammomys obesus model. $J$ Inflamm Res 7: 151-158.

20. Zigmond, E., S. W. Zangen, and O. Pappo, et al. 2009. Beta-glycosphingolipids improve glucose intolerance and hepatic steatosis of the Cohen diabetic rat. Am J Physiol Endocrinol Metab 296: E72-E78.

21. Zigmond, E., Z. Shalev, and O. Pappo, et al. 2008. NKT lymphocyte polarization determined by microenvironment signaling: a role for CD8 + lymphocytes and beta-glycosphingolipids. $J$ Autoimmun 31: 188-195.

22. Lalazar, G., A. Ben Ya'acov, and N. Eliakim-Raz, et al. 2008. Beta-glycosphingolipids-mediated lipid raft alteration is associated with redistribution of NKT cells and increased intrahepatic CD8 + T lymphocyte trapping. J Lipid Res 49: 1884-1893.

23. Lalazar, G., E. Zigmond, and S. Weksler-Zangen, et al. 2017. Oral Administration of betaGlucosylceramide for the Treatment of Insulin Resistance and Nonalcoholic Steatohepatitis: Results of a Double-Blind, Placebo-Controlled Trial. J Med Food 20: 458-464.

24. Margalit, M., S. Abu Gazala, and R. Alper, et al. 2005. Glucocerebroside treatment ameliorates ConA hepatitis by inhibition of NKT lymphocytes. Am J Physiol Gastrointest Liver Physio/289: G917G925.

25. Ilan, Y. 2016. Review article: novel methods for the treatment of non-alcoholic steatohepatitis targeting the gut immune system to decrease the systemic inflammatory response without immune suppression. Aliment Pharmacol Ther 44: 1168-1182.

26. Ilan, Y. 2016. Compounds of the sphingomyelin-ceramide-glycosphingolipid pathways as secondary messenger molecules: new targets for novel therapies for fatty liver disease and insulin resistance. Am J Physiol Gastrointest Liver Physio/310: G1102-G1117.

27. Ilan, Y. 2019. beta-Glycosphingolipids as Mediators of Both Inflammation and Immune Tolerance: A Manifestation of Randomness in Biological Systems. Front Immunol 10: 1143.

28. Adar, T., R. Shankar Lankalapalli, and R. Bittman, et al. 2020. The assembly of glycosphingolipid determines their immunomodulatory effect: A novel method for structure-based design of immunotherapy. Cell Immunol 355: 104157.

29. Folch, J., M. Lees, and G. H. Sloane Stanley. 1957. A simple method for the isolation and purification of total lipides from animal tissues. J Biol Chem 226: 497-509. 
30. Federico, A., E. D'Aiuto, and F. Borriello, et al. 2010. Fat: a matter of disturbance for the immune system. World J Gastroenterol 16: 4762-4772.

31. Pelleymounter, M. A., M. J. Cullen, and M. B. Baker, et al. 1995. Effects of the obese gene product on body weight regulation in ob/ob mice. Science 269: 540-543.

32. Ilan, Y., R. Maron, and A. M. Tukpah, et al. 2010. Induction of regulatory T cells decreases adipose inflammation and alleviates insulin resistance in ob/ob mice. Proc Natl Acad Sci U SA 107: 97659770.

33. Margalit, M., Z. Shalev, and O. Pappo, et al. 2006. Glucocerebroside ameliorates the metabolic syndrome in OB/OB mice. J Pharmacol Exp Ther 319: 105-110.

34. Elinav, E., O. Pappo, and M. Sklair-Levy, et al. 2006. Adoptive transfer of regulatory NKT lymphocytes ameliorates non-alcoholic steatohepatitis and glucose intolerance in ob/ob mice and is associated with intrahepatic CD8 trapping. J Patho/ 209: 121-128.

35. Guebre-Xabier, M., S. Yang, and H. Z. Lin, et al. Altered hepatic lymphocyte subpopulations in obesityrelated murine fatty livers: potential mechanism for sensitization to liver damage. Hepatology 2000;31:633 - 40 .

36. Godfrey, D. I., H. R. MacDonald, and M. Kronenberg, et al. 2004. NKT cells: what's in a name? Nat Rev Immunol 4: 231-237.

37. Li, Z., M. J. Soloski, and A. M. Diehl. 2005. Dietary factors alter hepatic innate immune system in mice with nonalcoholic fatty liver disease. Hepatology 42: 880-885.

38. Black, B. L., J. Croom, and E. J. Eisen, et al. 1998. Differential effects of fat and sucrose on body composition in A/J and C57BL/6 mice. Metabolism 47: 1354-1359.

39. Li, Z., H. Lin, and S. Yang, et al. 2002. Murine leptin deficiency alters Kupffer cell production of cytokines that regulate the innate immune system. Gastroenterology 123: 1304-1310.

40. Schmieg, J., G. Yang, and R. W. Franck, et al. 2005. Glycolipid presentation to natural killer T cells differs in an organ-dependent fashion. Proc Natl Acad Sci U S A 102: 1127-1132.

41. Hua, J., X. Ma, and T. Webb, et al. 2010. Dietary fatty acids modulate antigen presentation to hepatic NKT cells in nonalcoholic fatty liver disease. J Lipid Res 51: 1696-1703.

42. Russo, L., and C. N. Lumeng. 2018. Properties and functions of adipose tissue macrophages in obesity. Immunology 155: 407-417.

43. Zhao, Y., L. Lin, and J. Li, et al. 2018. CD4(+) T cells in obesity and obesity-associated diseases. Cell Immunol 332: 1-6.

44. Tang, J. T., and Y. M. Mao. 2017. Pharmacotherapy of nonalcoholic steatohepatitis: Reflections on the existing evidence. J Dig Dis 18: 607-617.

45. Das, K., and A. Chowdhury. 2013. Lean NASH: distinctiveness and clinical implication. Hepatol Int 7 (Suppl 2): 806-813.

46. Araujo, A. R., N. Rosso, and G. Bedogni, et al. 2018. Global epidemiology of non-alcoholic fatty liver disease/non-alcoholic steatohepatitis: What we need in the future. Liver Int 38 (Suppl 1): 47-51. 
47. Ilan, Y. Immune rebalancing by oral immunotherapy: A novel method for getting the immune system back on track. J Leukoc Biol 2018.

48. Zigmond, E., S. Preston, and O. Pappo, et al. 2007. Beta-glucosylceramide: a novel method for enhancement of natural killer T lymphoycte plasticity in murine models of immune-mediated disorders. Gut 56: 82-89.

49. Ilan, Y., M. Ohana, and O. Pappo, et al. 2007. Alleviation of acute and chronic graft-versus-host disease in a murine model is associated with glucocerebroside-enhanced natural killer T lymphocyte plasticity. Transplantation 83: 458-467.

50. Zhu, S., H. Zhang, and L. Bai. 2018. NKT cells in liver diseases. Front Med 12: 249-261.

51. Nishioka, Y., S. Masuda, and U. Tomaru, et al. 2018. CD1d-Restricted Type II NKT Cells Reactive With Endogenous Hydrophobic Peptides. Front Immunol 9: 548.

52. Brutkiewicz, R. R., L. Yunes-Medina, and J. Liu. 2018. Immune evasion of the CD1d/NKT cell axis. Curr Opin Immunol 52: 87-92.

53. Gill, P. S., A. Regmi, and P. A. Porter-Gill, et al. 1998. Interleukin-1 regulation of corticotropin-releasing factor (CRF), glucocorticoid receptor, c-fos and c-jun messenger RNA in the NPLC-KC cell line. Mol Cell Endocrinol 137: 31-39.

54. Rabelo, F., C. P. Oliveira, and J. Faintuch, et al. 2010. Pro- and anti-inflammatory cytokines in steatosis and steatohepatitis. Obes Surg 20: 906-912.

55. Tang, Y., Z. Bian, and L. Zhao, et al. 2011. Interleukin-17 exacerbates hepatic steatosis and inflammation in non-alcoholic fatty liver disease. Clin Exp Immunol 166: 281-290.

56. Ilan, Y. 2018. Analogy between non-alcoholic steatohepatitis (NASH) and hypertension: a stepwise patient-tailored approach for NASH treatment. Ann Gastroentero/ 31: 296-304.

\section{Figures}



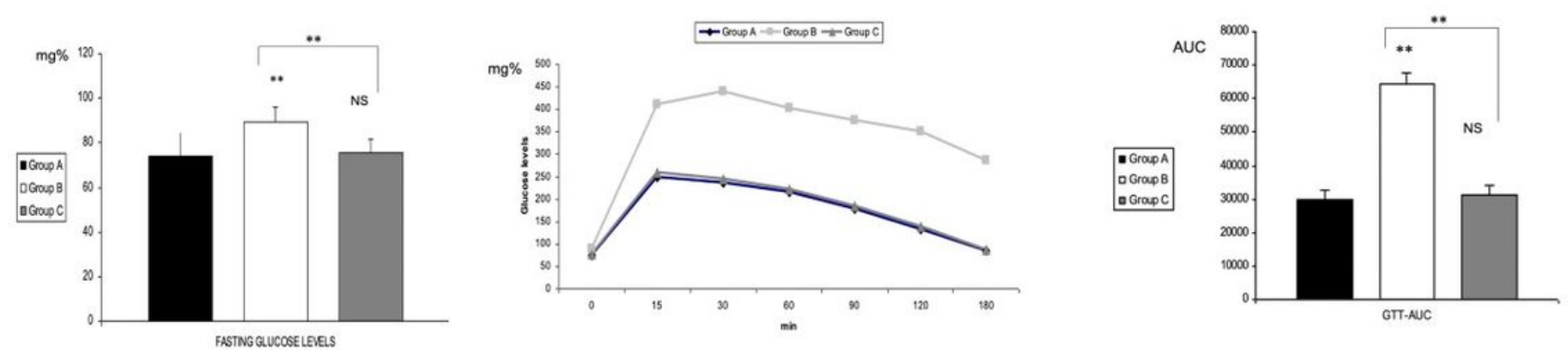

Figure 1A

Figure 1B

Figure 1C
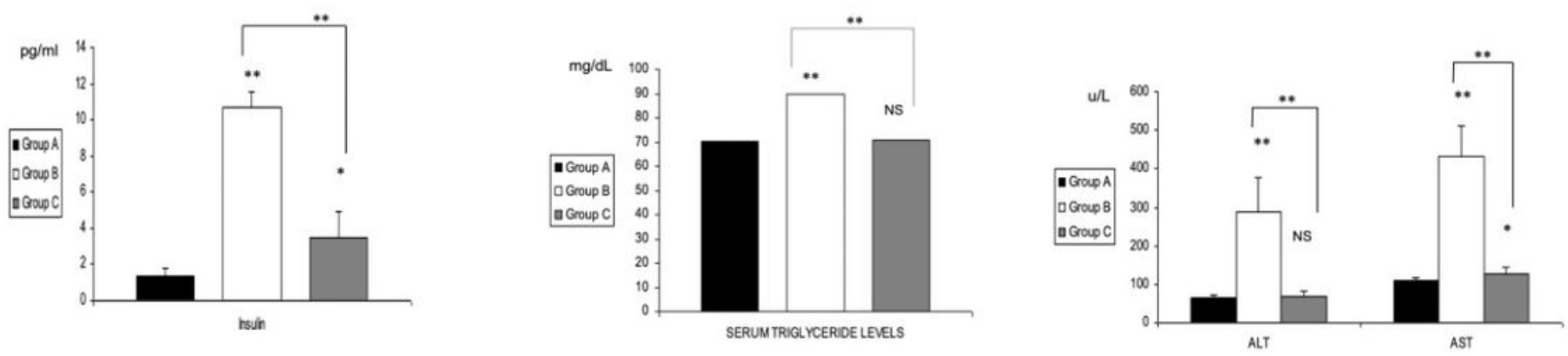

Figure 1D

Figure 1E

Figure 1F

\section{Figure 1}

Effect of steroid treatment on metabolic and liver parameters. Three groups of C57BL/6 mice were followed for eight weeks for assessment of the preventive effect of GC on the development of steroidmediated liver damage. Group A received vehicle alone; Group B received Dexamethasone; and group C received a combination of Dexamethasone with GC. Mice were followed for fasting serum glucose levels (A); postprandial glucose levels as manifested by glucose tolerance test absolute values (B) and AUC (C); serum insulin levels (D); serum triglyceride levels (E); and AST and ALT liver enzymes $(F) .\left({ }^{* \star} p<0.01\right.$, * $p<0.05)$. 

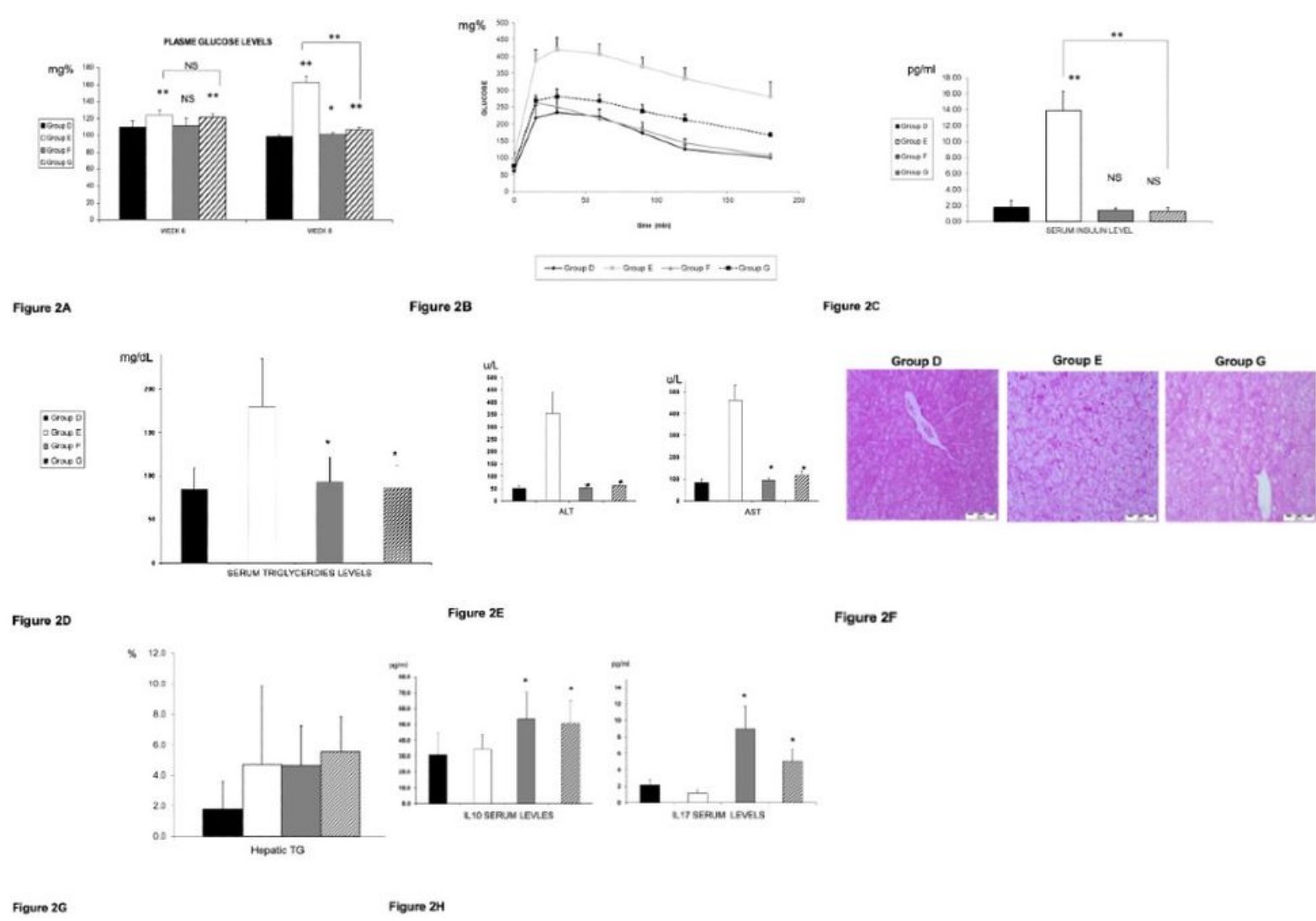

\section{Figure 2}

The therapeutic effect of GC on an existing metabolic and liver damage was studied in four groups of $\mathrm{C} 57 \mathrm{BL} / 6$ mice. Mice in group $\mathrm{D}$ received the vehicle alone; Group $\mathrm{E}$ received Dexamethasone; and group $\mathrm{F}$ received a combination of Dexamethasone with GC; mice in group G received Dexamethasone for 6 weeks followed by two weeks of Dexamethasone with GC. Mice were followed for fasting glucose levels (A), postprandial glucose levels as manifested by glucose tolerance test (B); serum insulin levels (C); serum triglyceride levels (D); AST and ALT liver enzymes (E); liver histology (H\&E X10) (F); hepatic triglycerides content $(G)$; and for serum levels of IL10 and IL17 (Figure H). $\left(* \star p<0.01,{ }^{\star} p<0.05\right)$. 

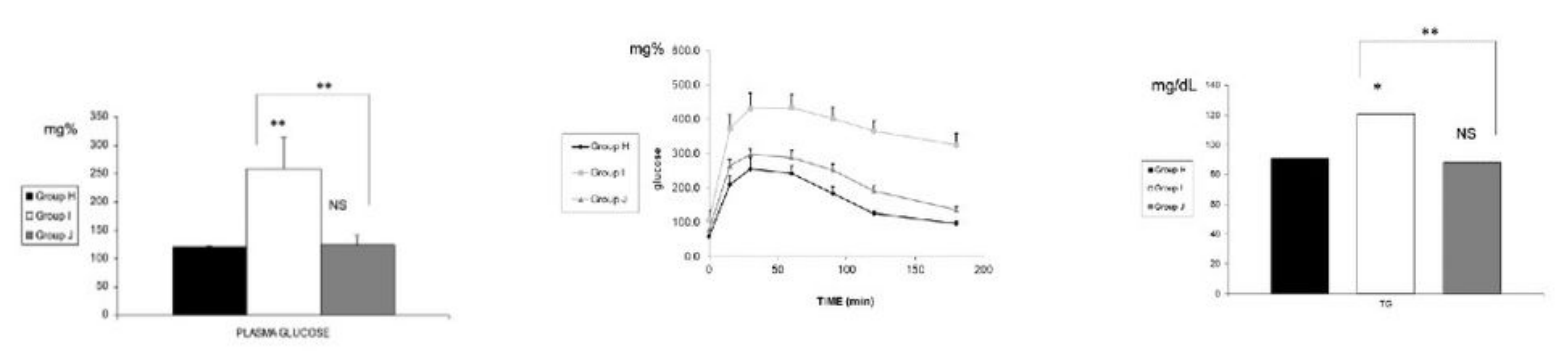

Figure 3A

Figure 3B

Figure 3C
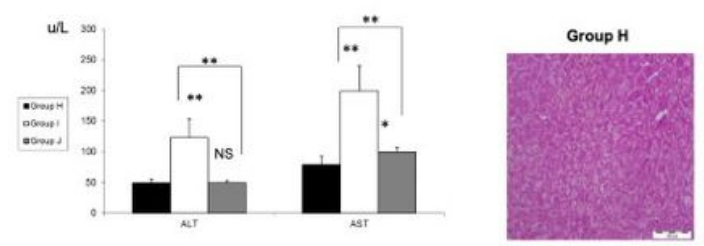

Group I
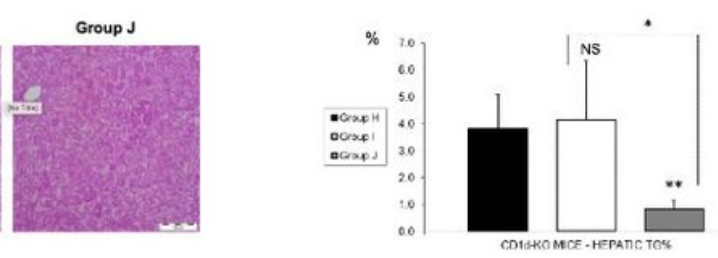

Figure 3D

Figure 3E

Figure $3 F$

\section{Figure 3}

The CD1d-dependency of the steroid-induced liver damage, and the steroid-protective effect of GC, were studied in three groups of $\mathrm{CD} 1 \mathrm{~d}-/$ - mice. Mice in group $\mathrm{H}$ received the vehicle alone; Group I received Dexamethasone; and Group J received a combination of Dexamethasone with GC for eight weeks. Mice were followed for fasting glucose levels (A), postprandial glucose levels as manifested by glucose tolerance test (B); serum triglyceride levels (C); AST and ALT liver enzymes (D); liver histology (H\&E X10) (E); and hepatic triglycerides content $(F) .(* * p<0.01, * p<0.05)$.

\section{Supplementary Files}

This is a list of supplementary files associated with this preprint. Click to download.

- Supplementaryfile.xls 